\title{
OXYGEN SENSOR PERFORMANCE EVALUATION USING TRANSIENT BOUNDARY CONDITIONS
}

\author{
André Bergel ${ }^{1}$, Nide Geraldo do Couto Ramos Fico Junior ${ }^{2}$ \\ ${ }^{1}$ General Motors Powertrain South America \\ ${ }^{2}$ Instituto Tecnológico da Aeronáutica \\ E-mails: andrebergel84@yahoo.com.br, nide.ficojr@gmail.com
}

\begin{abstract}
The purpose of this paper is to analysis the performance of an oxygen sensor using computational fluid dynamics (STAR-CD), and is based on scalars concentration around sensor element and static/dynamic performance data of oxygen sensor. The analysis uses transient boundary conditions, supplied by GT-Power software to approximate the real life utilization, and various velocities and loads were run to obtain the overall oxygen sensor dynamic performance. In this study, was analyzed the scalar concentration from each cylinder around the element sensor, simulating the oxygen sensor dynamic response outputs.
\end{abstract}

\section{INTRODUCTION}

The electronic fuel injection control system is a very important method to save fuel in the modern cars, and oxygen sensor is the most important piece of them. Oxygen sensor is responsible for providing information about air-fuel ratio (AFR) of exhaust gas, resulting from in-cylinder combustion process. The oxygen sensor provides information about the lambda value $(\lambda)$ that is calculated by:

$\lambda=\frac{A F R_{\text {Real }}}{A F R_{\text {Stoichiometric }}}$

This value is used to correct the amount of fuel that is injected in intake runner, to keep the mixture close to stoichiometry. A stoichiometric mixture is important to save fuel and achieve the best performance of a three-way catalyst converter.

Oxygen sensor signal is a switching type (nonlinear, only High/Low response), as can be view in Fig. 1, and the fuel control system is a switch type which tries to keep the AFR between slightly rich and lean. Figure 1 also presents samples of oxygen sensor signal without AFR imbalance between cylinder, and with one cylinder 10, 20 and $40 \%$ rich, to show the influence on signal output to electronic control unit.

When there is any cylinder with AFR imbalance, the signal will be a square wave coupled with high frequency oscillation. The period of the high frequency oscillation is the engine 720 deg-cycle time and the oscillation amplitude is related to the severity of the cylinder AFR imbalance. 


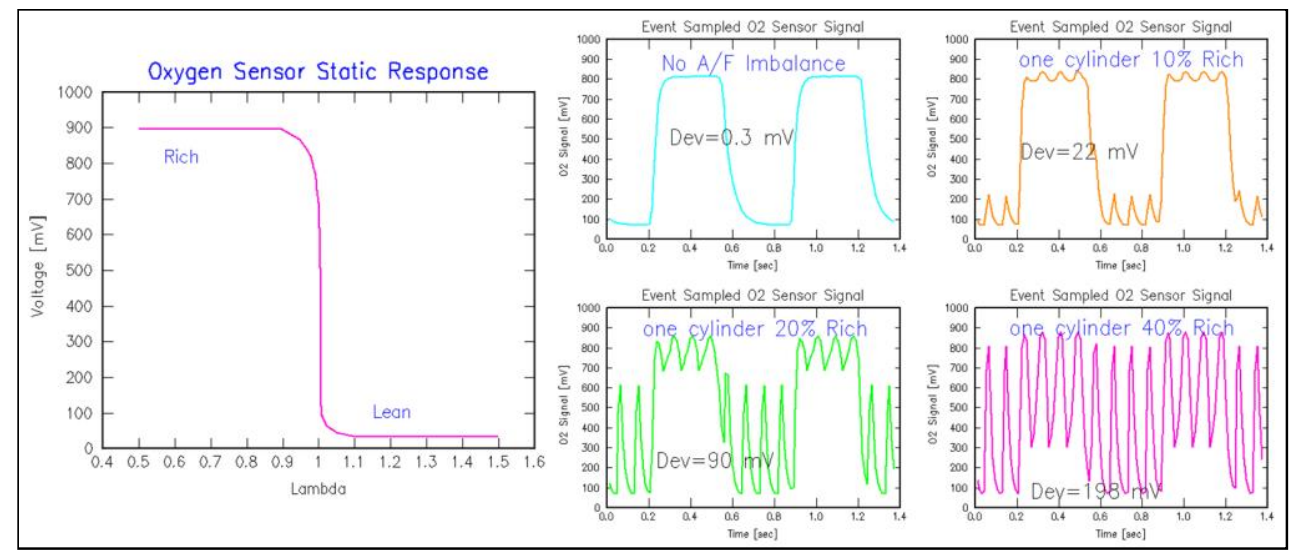

Figure 1. Examples of oxygen sensor static response and output sygnal with cylinder AFR imbalance.

\section{METODOLOGY}

In order to running these analysis, were needed first the transient boundary conditions, which are obtained by a GT-Power model of the engine. The data acquisition is not interesting for this study so, just an example will be showed in Fig. 2, for a case, to show the variables time histories vs. crank angle degree (CAD), for a full cycle (720 CAD). The variables required by STAR-CD to apply transient boundary conditions are: pressure, temperature, density and mass flow rate, for all runners and the outlet of the catalytic converter.

The cases studied in this analysis were obtained by varying engine speed, between 800 and $3200 \mathrm{rpm}$ (revolutions per minute) and load, between 100 and 250 APC (air per cylinder, in $\mathrm{mg}$ ), and all cases are showed in the Table 1.

In STAR-CD software, the mesh was imported from ICEMCFD v12 software. This mesh is almost all hexahedral, excepting the mesh around $\mathrm{O} 2$ sensor that is tetrahedral. This mesh has an extrusion on inlet runners, to develop the boundary layer. Geometry, mesh, sensor covers and elements around sensor, used to calculate the scalars concentrations from each cylinder, are presented in Fig. 3.

Table 1. All case numbers and conditions runned on analysis.

\begin{tabular}{|c|c|c|c|c|}
\hline \multirow{2}{*}{ APC (mg) } & \multicolumn{4}{|c|}{ Engine Speed (rpm) } \\
\cline { 2 - 5 } & $\mathbf{8 0 0}$ & $\mathbf{1 4 0 0}$ & $\mathbf{2 0 0 0}$ & $\mathbf{3 2 0 0}$ \\
\hline 100 & Case 1 & Case 4 & Case 7 & Case 10 \\
\hline 175 & Case 2 & Case 5 & Case 8 & Case 11 \\
\hline 250 & Case 3 & Case 6 & Case 9 & Case 12 \\
\hline
\end{tabular}

To set the fluid features, was used hot air (ideal gas), and was set a scalar for each inlet runner, to identify the gas from each cylinder, and the analysis to be able to calculate conversion efficiencies and flow uniformities. The turbulence model used was the $\kappa-\varepsilon$ model at default values. 


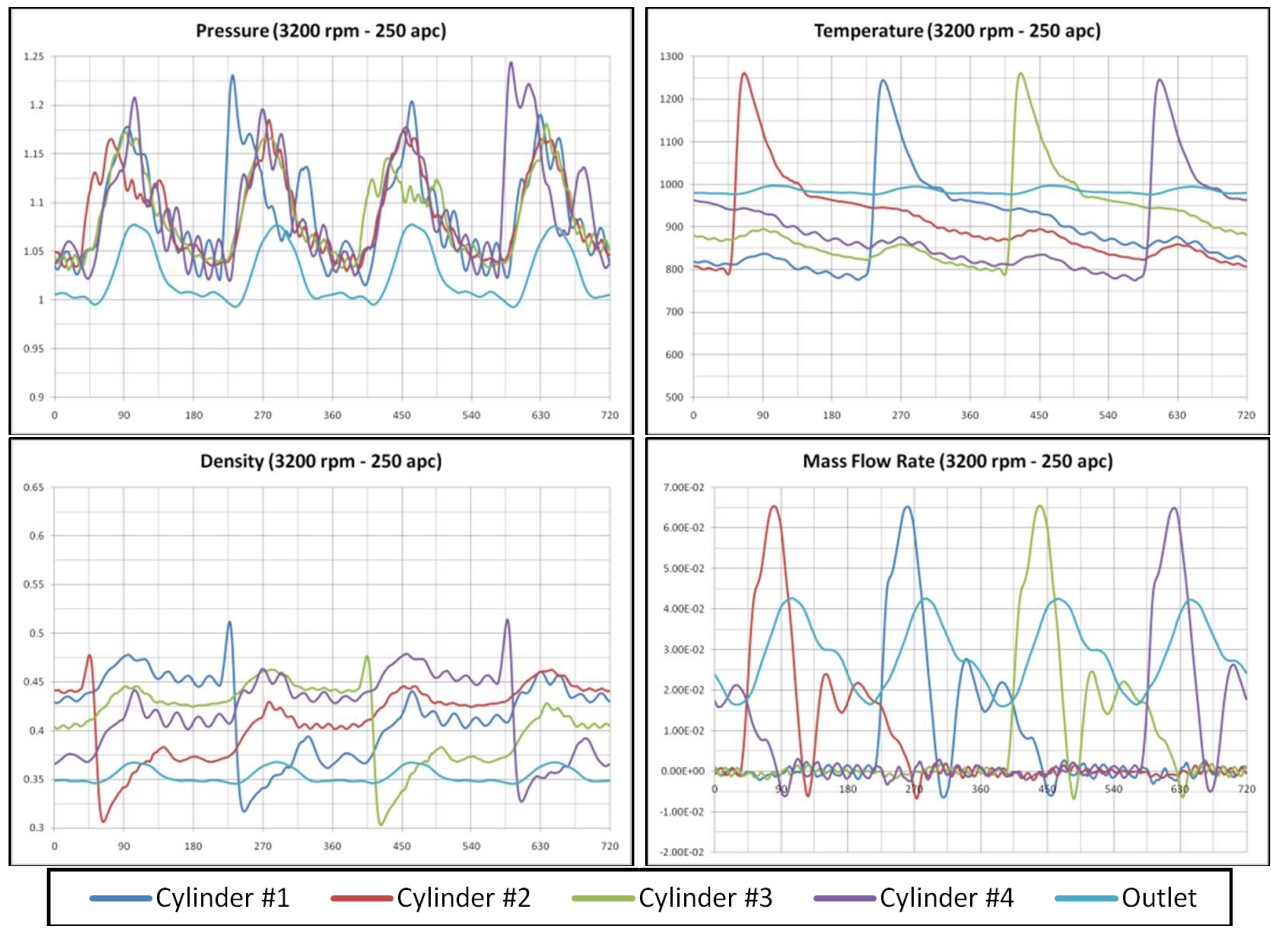

Figure 2. Example of the boundary conditions used on the analysis, for case of $3200 \mathrm{rpm}$ and 250 apc.

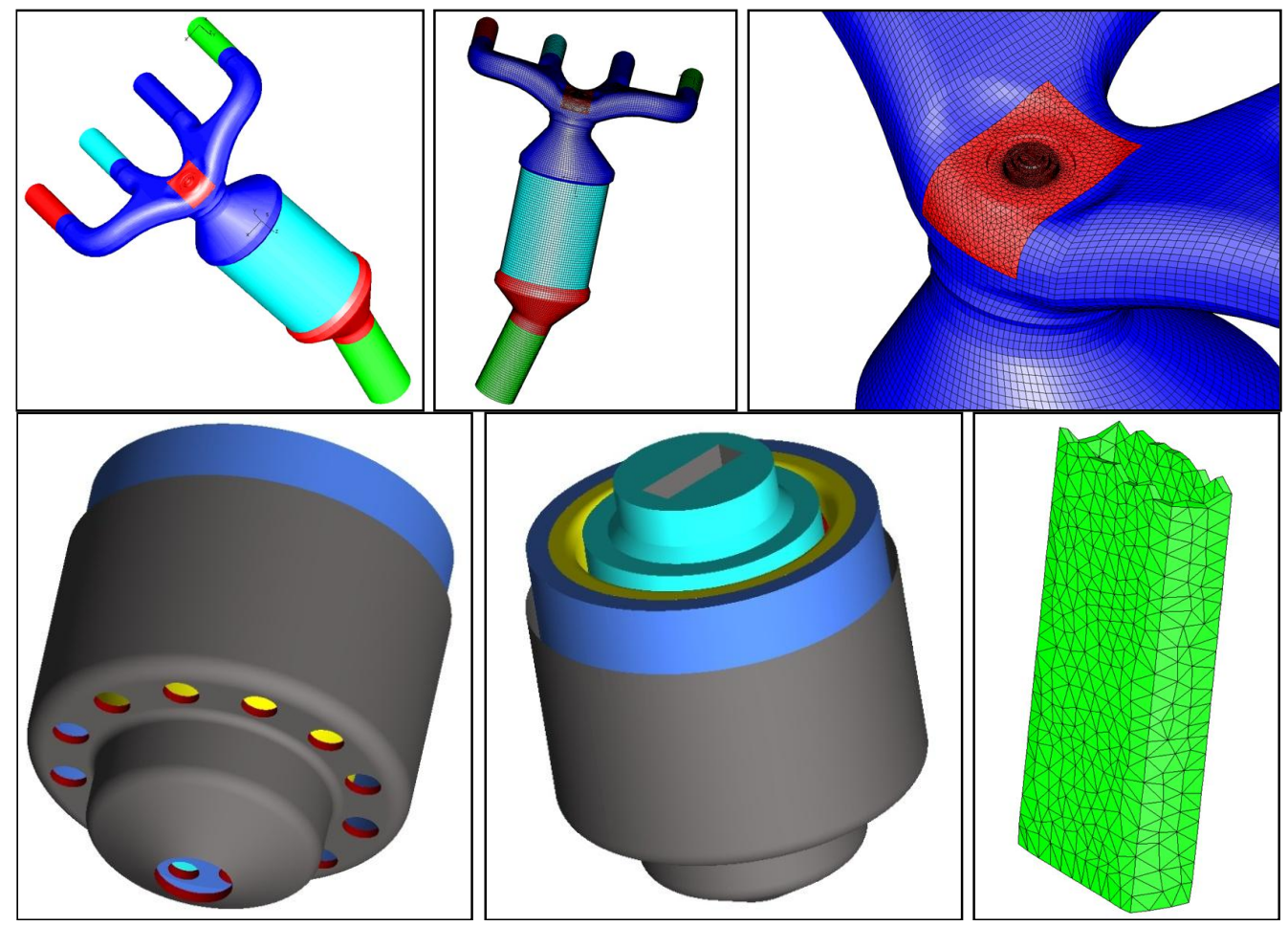

Figure 3. Geometry, mesh and oxygen sensor details and covers, and prismatic elements around element sensor used on analysis. 
The analysis boundary conditions for walls are fixed heat transfer, with a temperature of 298 $\mathrm{K}$ and a value of $0.1 \mathrm{~m}^{2} \mathrm{~K} / \mathrm{W}$ to the resistance. In the catalytic converter, an adiabatic wall was used to simulate the insulation.

To simulate the pressure drop for the substrate, was used a porous media to represent it. A program provides alpha and beta values to STAR-CD, from data for substrate shape of the honeycomb, length, frontal area, washcoat thickness, wall thickness and mean temperature and pressure.

The analysis run by 6 cycles of $720 \mathrm{CAD}$, the 5 initial cycles are run to obtain a periodically fully-developed flow, and data was collected just on $6^{\text {th }}$ cycle. The timestep used on analysis corresponds to $1 \mathrm{CAD}$ revolution.

\section{RESULTS}

Results analysis is taken based on from-cylinder concentration calculated around element sensor, averaged by prismatic mesh elements, showed in Figure 3. We use on analysis two metrics: From-cylinder-I Concentration Curve Deviation ( $\left.\mathrm{D}_{\mathrm{I}}\right)$, deviation of cylinder I concentration curve, and Short Term Deviation (STD), deviation of oxygen sensor signal. In Figure 4 can be view an example of average values and imbalances taken into account.
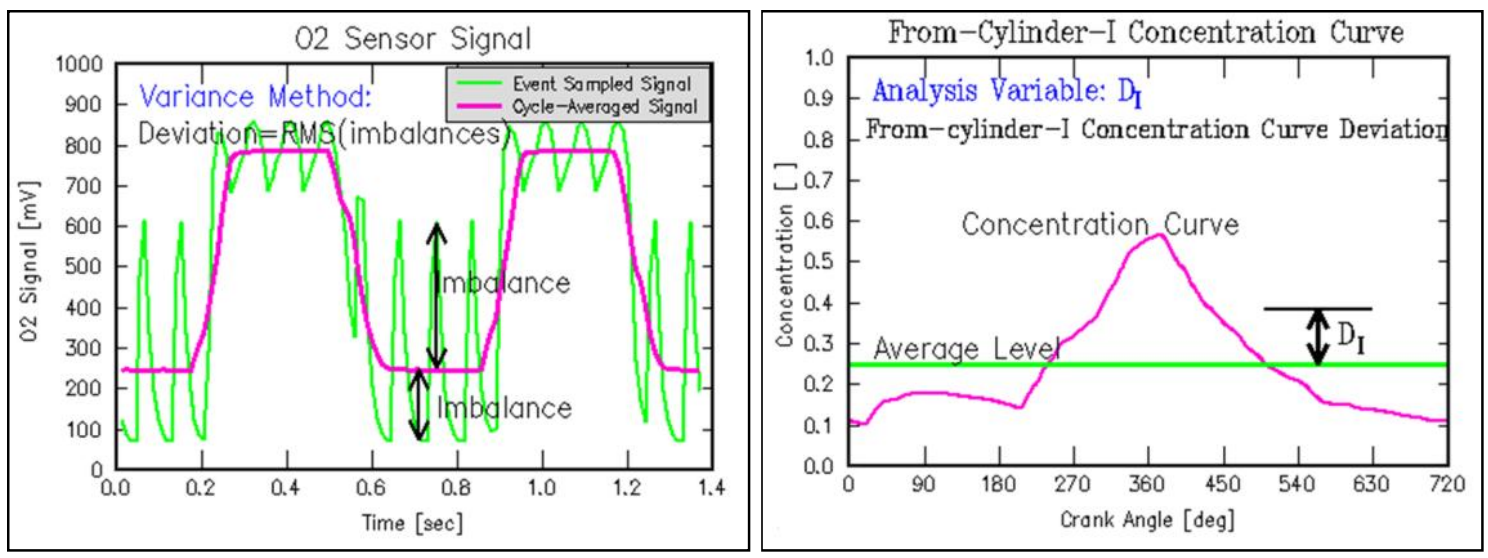

Figure 4. STD and Curve Deviation examples, showing the average values and imbalances.

This approach, basically, gets rid of the unwanted low frequency signal created by rich/lean switching in fuel control and the STD value are related to the cylinder AFR imbalance only. For the same amount of cylinder AFR imbalance, if oxygen sensor can get higher $\mathrm{D}_{\mathrm{I}}$ value then the measured STD value will be large enough for detection. If the DI value is very low (flat curve, no dynamic up-and-down), even though the cylinder AFR is totally out of balance, still cannot detect it because STD will be very low. Good or bad output signal from oxygen sensor can be related to good/bad sensor type or location. An example for booth can be view in Figure 5. 


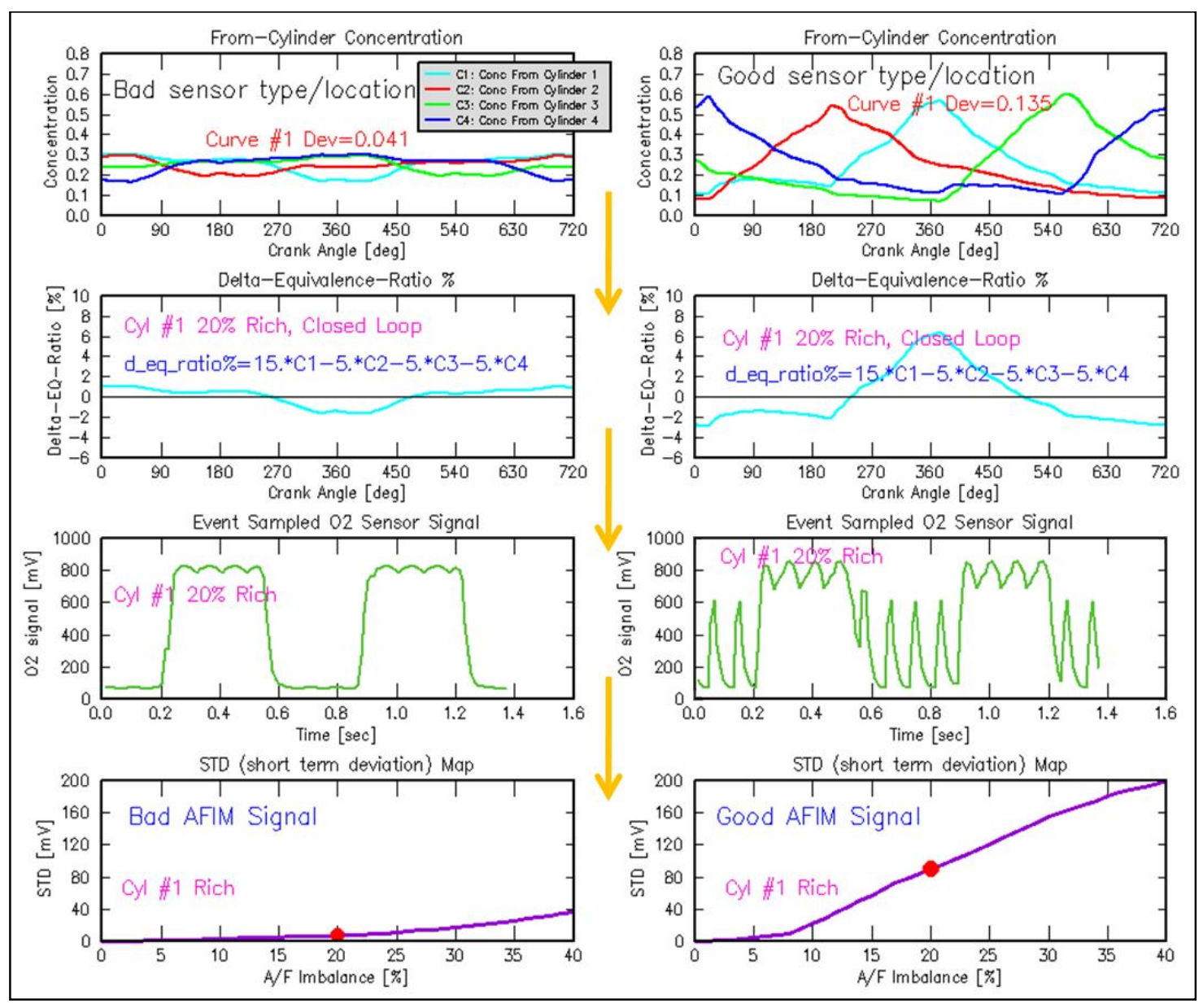

Figure 5. Relation between oxygen sensor concentration curve and STD Map, showing bad and good sensor type or location.

\subsection{From-Cylinder Concentration Curve and Curve Deviation}

The concentration curves are show in Fig. 6. The from-cylinder concentration is volume averaged around oxygen sensor prismatic elements show in Fig. 3. All plots are from-cylinder concentration vs. CAD.

These results show that oxygen sensor cannot see cylinders evenly, and it is a problem to identify which cylinder is rich or lean. There are no well defined peaks or valleys corresponding to cylinder pulsation, like showed in Fig. 5. By this results, can be inferred that oxygen sensor signal output, Short Term Deviation and AFIM Deviation Ratio (ADR), will have bad results too. 


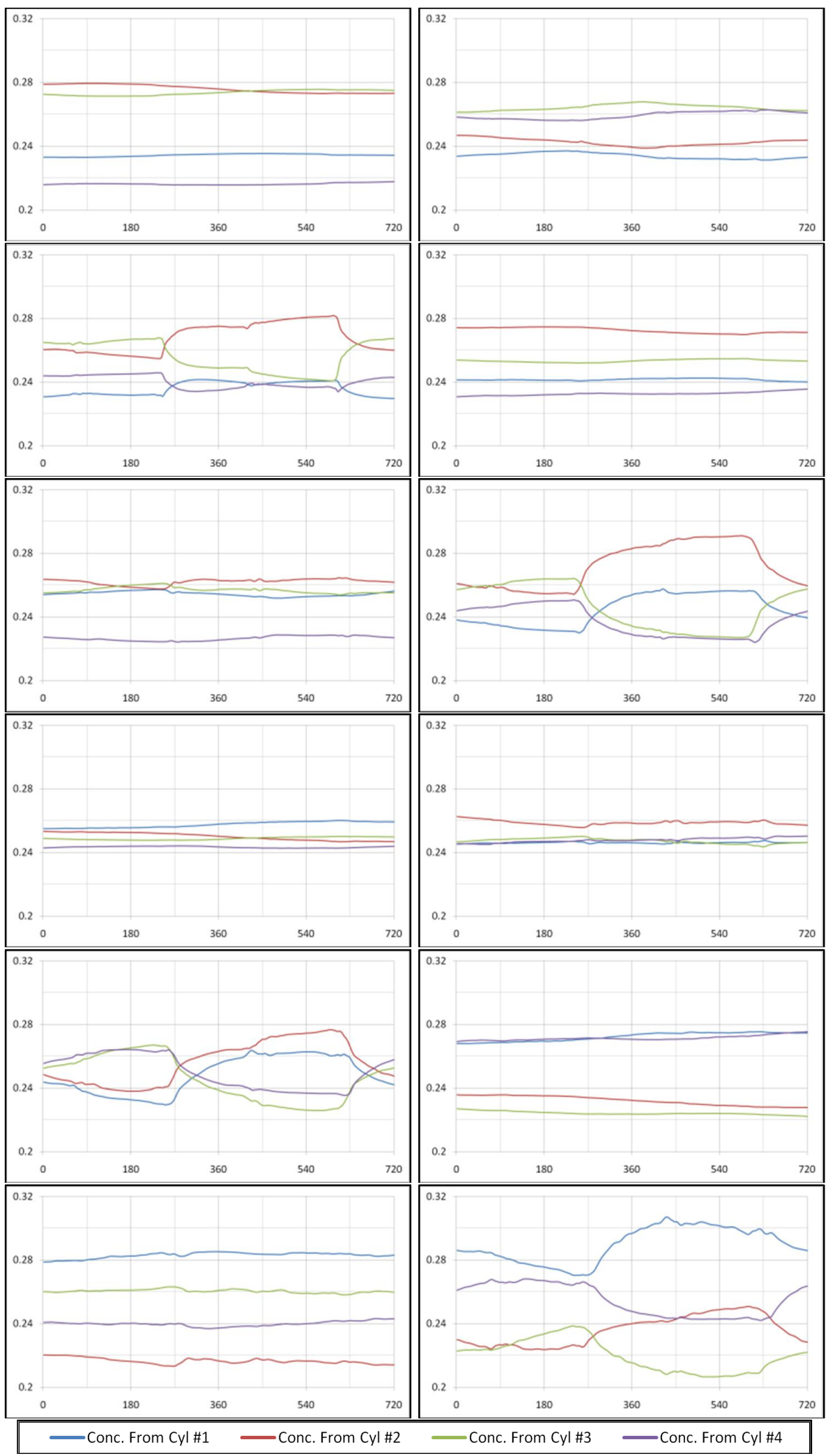

Figure 6. From-cylinder concentration curves for all cases. 
Figure 7 shows from-cylinder curve deviation for all cases. The from-cylinder curve deviation indicates the dispersion from mean value and should have values in order of 0.05 to 0.1 . To calculate the curve deviation $\left(D_{I}\right)$ was used the following formula:

$D_{I}=\sqrt{\frac{1}{N} \sum_{i=1}^{N}\left(C_{I}(i)-\bar{C}_{I}\right)^{2}}$

Where $C_{I}(i)$ is value at point $i$ of the From-cylinder-I concentration curve, $\bar{C}_{I}$ is the average value of the From-cylinder-I concentration curve and $N$ is the total number of data points in the curve.

The deviation values are lower than the expected range (between 0.05 and 0.1 ), these results agree with flat concentration curves show in Fig. 6. It means that has little dispersion from the average value and the oxygen sensor do not have dynamic response, due to exhaust manifold geometric features or sensor positioning, and the output signal to Electronic Control Unit (ECU) will be a square wave without significant imbalances, preventing ECU to correct rich air-fuel mixture from any cylinder.
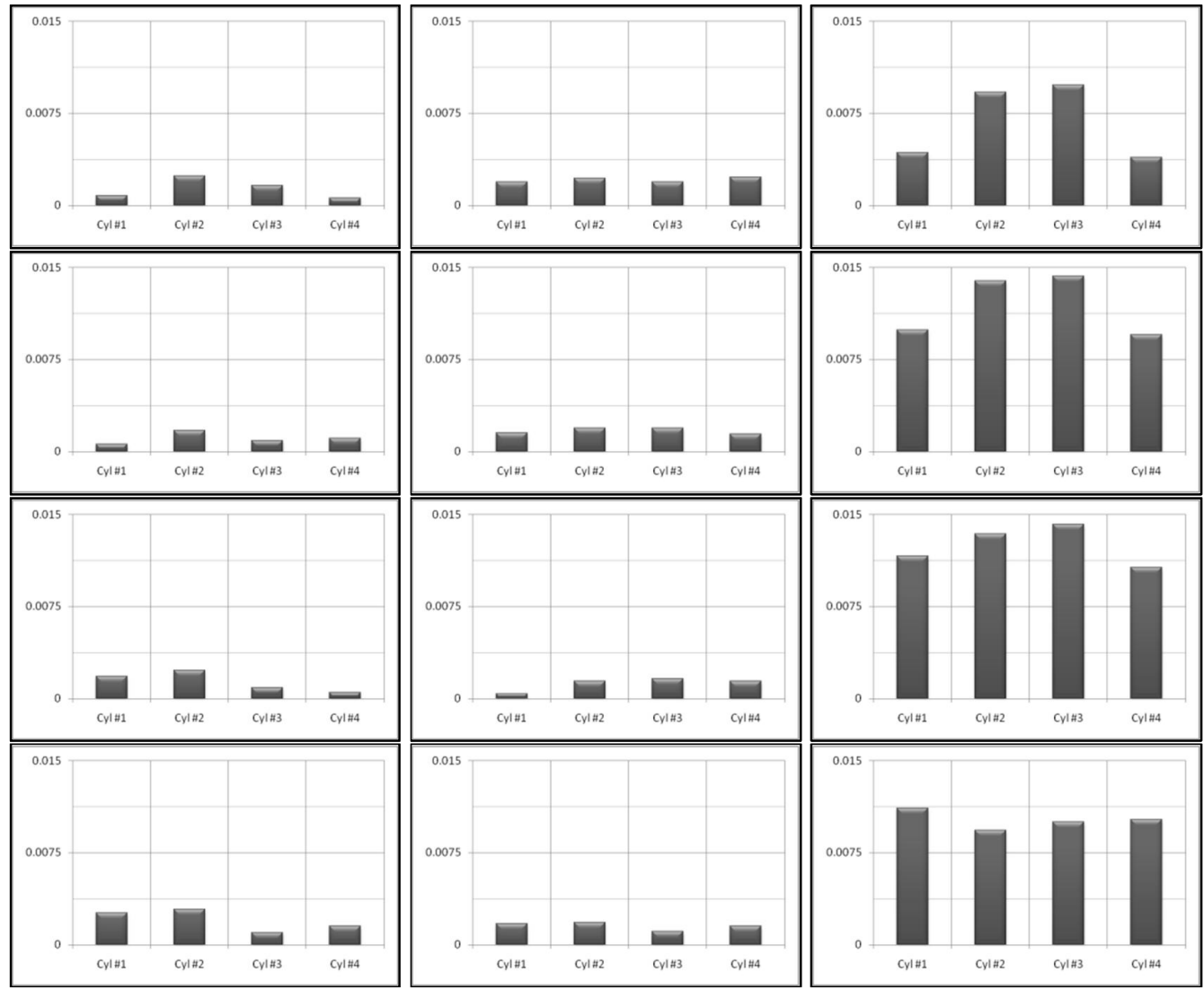

Figure 7. From-cylinder curve standard deviation for all cases.

\subsection{Short Term Deviation Map and Oxygen Sensor Output Examples}


For the optimal oxygen sensor location for AFIM, the sensor has to be put at a location where it has the highest STD output for the cylinder that has the lowest STD output among all cylinders in the bank for a given cylinder AFR imbalance. Figure 8 shows the STD map versus imbalance percentage. The STD formula is given on Eq. 3 .

$S T D=\sqrt{\frac{1}{N} \sum_{i=1}^{N}\left(S_{i}-\bar{S}\right)^{2}}$

Where $S_{i}$ is the event sampled oxygen sensor signal, in $[\mathrm{mV}], \bar{S}$ is the oxygen sensor engine-cycle-averaged signal, in $[\mathrm{mV}]$ and $N$ is the total number of samplings in a short term period.

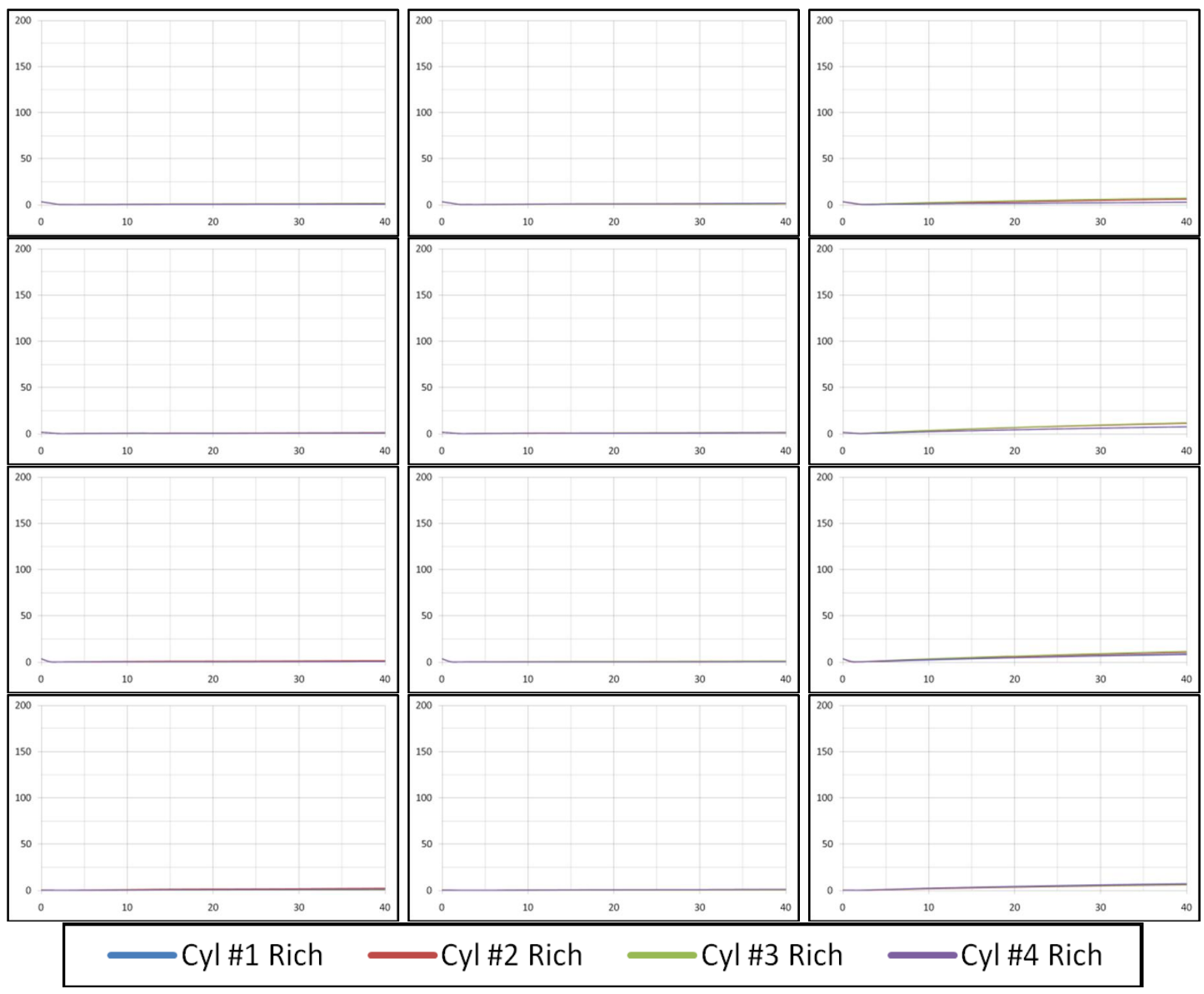

Figure 8. Short Term Deviation map for all cases.

The STD map figures show that oxygen sensor has not able to identify differences in from-cylinder concentration evenly. There are no visible differences between cylinders or so long the imbalance increases. Based on these results can be inferred that oxygen sensor output signal will show just the square wave without the high oscillation frequency imbalances from cylinder richness or poorness.

To estimate the oxygen sensor signal output, were used static and dynamic responses data, presented on Fig. 9. This data is provided by the supplier and correspond just to 
the element sensor, because the shielding effect of the cover was taken into account in geometry discretization. The evaluation for oxygen sensor signal output is show in Fig. 10, and all graphs are in units of seconds vs. $\mathrm{mV}$.

Can be noted that oxygen sensor output signal are square waves without significant imbalances, as inferred before. These results are bad because the ECU will not be able to identify which cylinder is rich, just mean value of AFR. It is dangerous because one cylinder can be $30 \%$ rich and others $10 \%$ lean, which is a bad situation, but average value will be a stoichiometric mixture and ECU will not correct it.
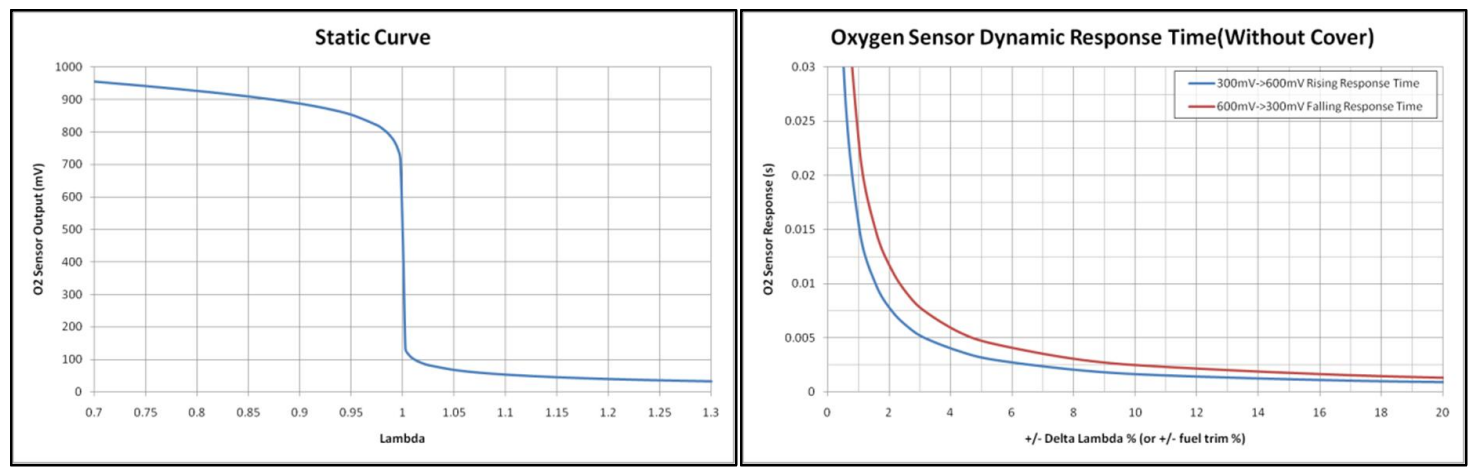

Figure 9. Static and dynamic data used to generate the oxygen sensor output sample.

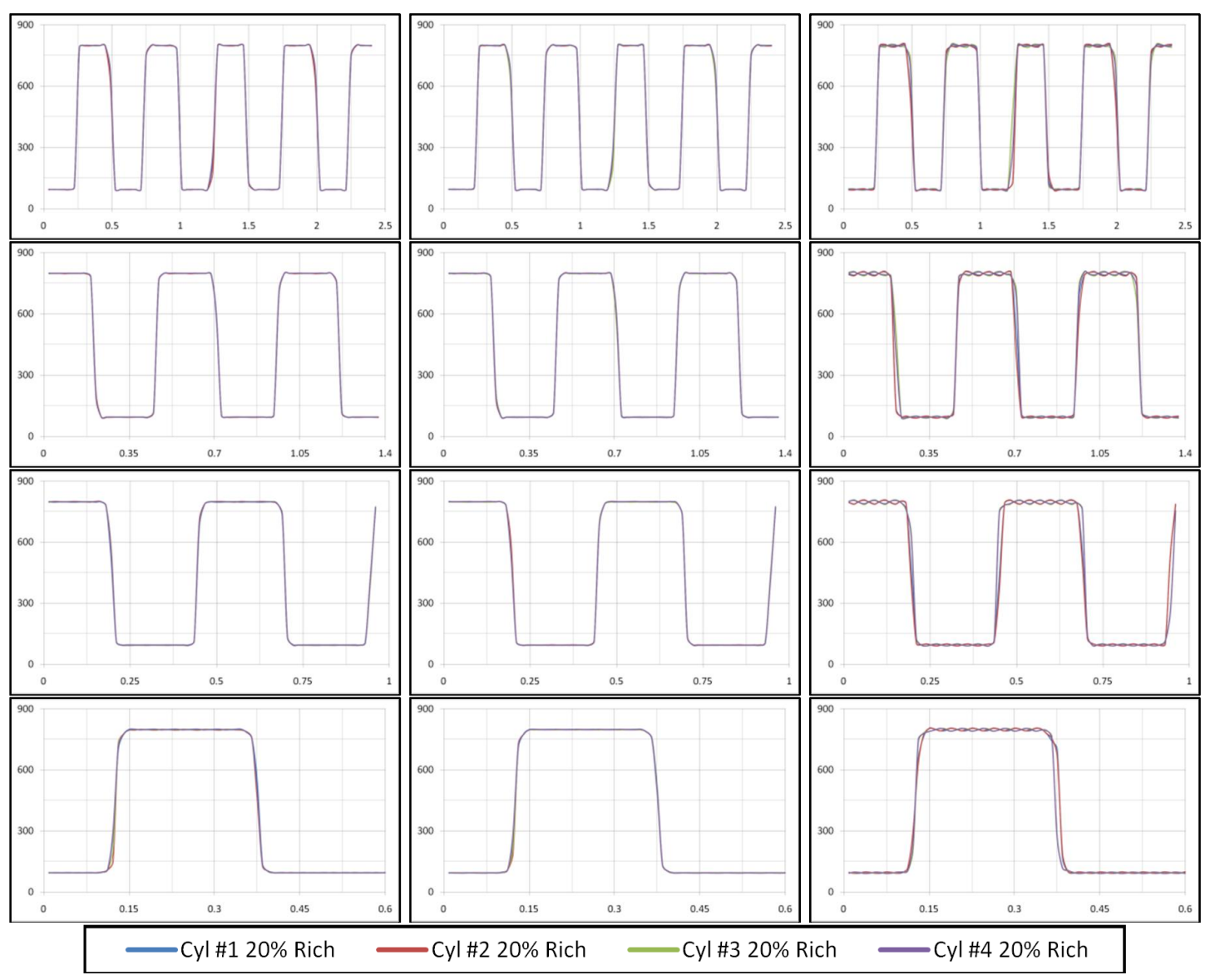


Figure 10. Oxygen sensor output examples for all cases.

2.3. AFIM Deviation Ratio, Relative Sensitivity Ratio and Trigger Imbalance Percentage

AFIM Deviation Ratio is a metric used to estimate cylinder imbalance influence on oxygen sensor signal output. To calculate this metric was used Eq. 4, and a graphic showing an example is in Fig. 11. ADR's values for all cases are show in Fig. 12. Higher ADR values (greater than 5), for all cylinders in $50 \%$ of cases at least, is a good result.

$A D R^{I}=\frac{S T D_{\text {cylinder } 40 \% \text { rich }}^{I}-S T D_{5} \% W P A}{S T D_{5} \% W P A}$

Where $S T D_{\text {cylinder } 40 \% \text { rich }}^{I}$ is the STD value with cylinder I $40 \%$ rich imbalance, $S T D_{5 \% W P A}$ is the highest STD value for any cylinder $5 \%$ rich or lean in the bank and $W P A$ is worst performing acceptable.

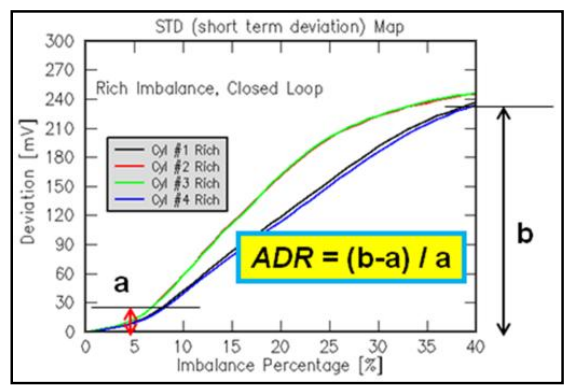

Figure 11. AFIM Deviation Ratio graphic example.

Relative Sensitivity Ratio (RSR) is a metric wich compare least significant STD at $40 \%$ rich imbalance of any cylinder and most significant STD at $20 \%$, rich or lean, imbalance for any cylinder, and is calculated by Eq. 5. Trigger Imbalance Percentage (TIP) is the imbalance percentage for any cylinder, rich or lean, to reach $L S D_{40 \% \text { rich }}$ level. Graphic examples for RSR and TIP are show on Fig. 13.

$R S R=\frac{L S D_{40 \% \text { rich }}}{M S D_{20 \% \text { rich or lean }}}$

Where $L S D$ is the Least Significant Deviation among cylinders in the bank and $M S D$ is the Most Significant Deviation among cylinders in the bank. 

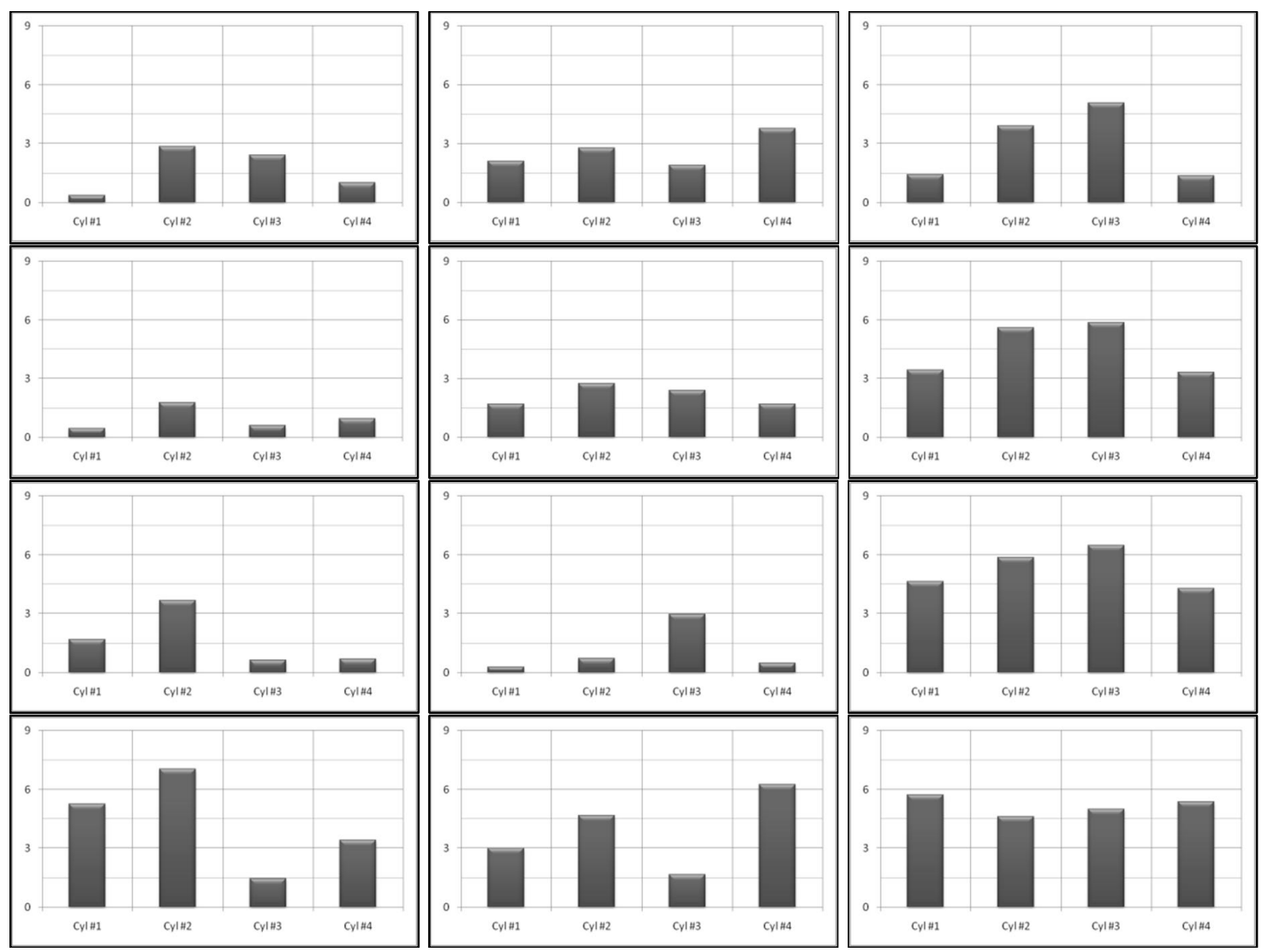

Figure 12. AFIM Deviation Ratios for all cases.

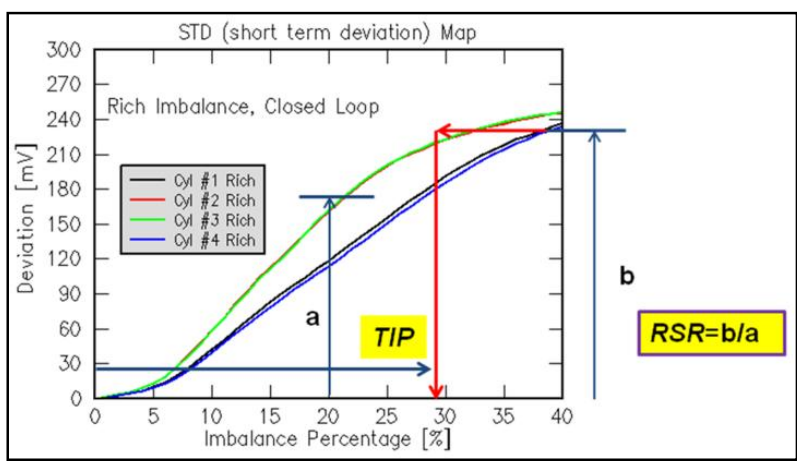

Figure 13. Relative Sensitivity Ratio and Trigger Imbalance Percentage graphic example for all cases.

These results are not good too, comparing to example show in Fig. 13. A RSR value of 1.5 would be good, showing that the STD value grow up as the imbalance increases. Results for TIP are bad too, because more than half of the cases have a zero value, showing that there is no significant growing of the STD values as the imbalance increases. 


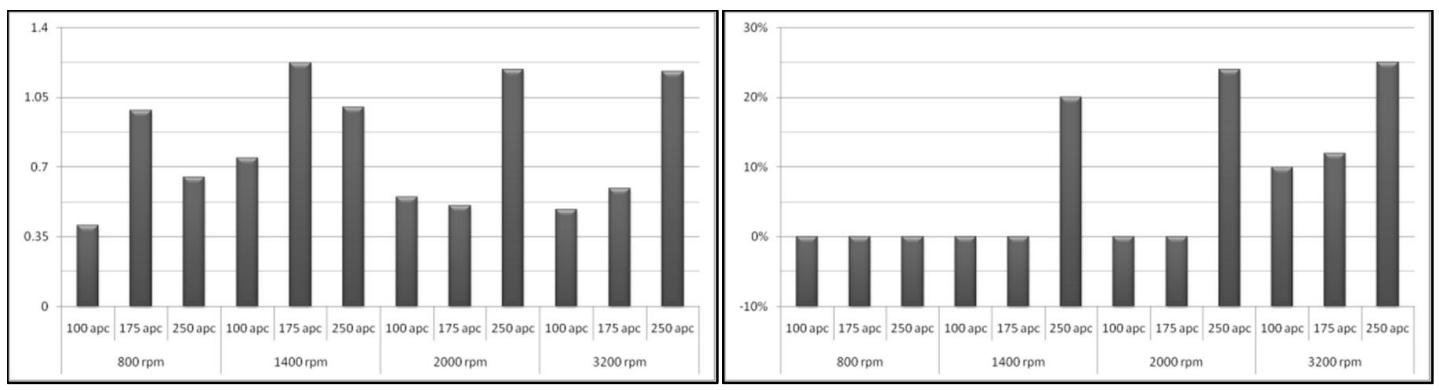

Figure 14. Relative Sensitivity Ratio and Trigger Imbalance Percentage for all cases.

\section{CONCLUSIONS}

The oxygen sensor position is very important in order to provide to electronic fuel injection system a good quality signal. A good sign is that which allows the identification of imbalances in the AFR amongst which cylinder is causing these imbalances. To perform this, the oxygen sensor must have dynamic response as fast as possible and should have covers not so much closed. The exhaust manifold geometry should have equal runner lengths and oxygen sensor must be putted on a local which can see all the cylinders evenly, preferably in a tube after runner's junction.

This analysis concluded that the actual oxygen sensor position is not meeting good results. Almost all of the from-cylinder concentration curves are flat or something close, resulting in very low from-cylinder curve deviations. These low values for from-cylinder curve deviations shows that the exhaust gas inside the oxygen sensor covers is not being renewed, and the value measured is not pulsating. These values, averaged and almost constant, make the ECU unable to identify which cylinder is rich or lean, so no correction is made in the overall AFR. One way to avoid this situation is using an oxygen sensor with a less restricting cover, like a

first approach. In the future, exhaust manifold can be redesigned to equalize runner length and relocate the oxygen sensor on the junction of them.

\section{ACKNOWLEDGMENTS}

To GMPT South America, for providing physical and virtual space for the analysis.

To Mitchell Ebbs, from GMPT North America, for supporting with his great knowledge and experience.

\section{REFERENCES}

[1] Heywood, John, Internal Combustion Engine Fundamentals, McGraw Hill, New York, NY, 1988.

[2] Schlichting, H., Boundary Layer Theory, $7^{\text {th }}$ Edition, McGraw Hill, New York, NY, 1979.

[3] ICEM CFD, ICEM CFD V4.10 Online Documentation, July, 2000.

[4] Computational Dynamics Limited, User Guide STARCD Version 3.10, 1999. 